\title{
217 Idiopathische entzündliche Myopathien
}

T. Voit

Definition. Einige Krankheiten der Muskulatur werden wegen ihres im Vordergrund stehenden entzündlichen Charakters als idiopathische entzündliche Myopathien zusammengefasst (s. folgende Übersicht). Obwohl in Einzelfällen infektiöse Erreger als Ursache oder Auslöser nachgewiesen werden können, stehen bei der Mehrzahl der Patienten Autoimmunmechanismen bei Auslösung und Unterhaltung der Krankheit im Vordergrund.

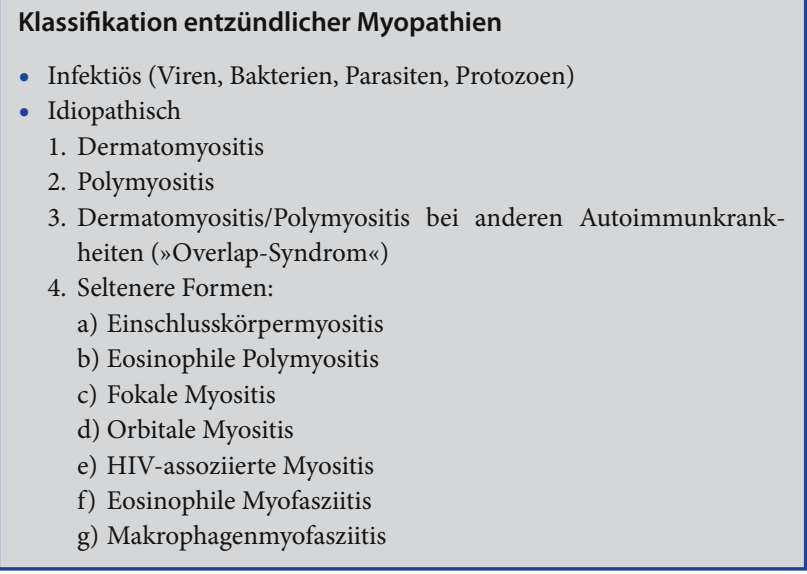

Epidemiologie. Die Inzidenz aller entzündlichen Myopathien beträgt 1:100.000. Dabei ist im Kindesalter die juvenile Dermatomyositis die bei weitem häufigste Form.

\section{Juvenile Dermatomyositis (DM) und Polymyositis (PM) \\ $(\triangleright$ Kap. 92 )}

\section{Einschlusskörpermyositis}

Die Einschlusskörpermyositis unterscheidet sich klinisch und histopathologisch von den anderen Formen idiopathisch-entzündlicher Myopathien. Klinisch steht eine langsam progrediente Schwäche im Vordergrund, die meist im Jugend- oder jungen Erwachsenenalter einsetzt und eine proximale oder distale Verteilung zeigen kann. Charakteristischerweise ist die Krankheit refraktär gegen Behandlung mit Steroiden, Immunsuppressiva oder i. v.-Immunglobulinen. Wie der Name sagt, ist sie histologisch durch Einschlusskörper gekennzeichnet, umrandete Vakuolen mit basophilem Inhalt in den Muskelfasern (rimmed vacuoles). Es wird zunehmend klar, dass zumindest ein substanzieller Anteil der Patienten mit Einschlusskörpermyositis an einer der genetisch bedingten distalen Myopathien ( $\bullet$ Kap. 214) leidet, bei denen ebenfalls derartige Vakuolen vorkommen. Immunzytochemische Untersuchungen wiesen darauf hin, dass einer Blockierung des Proteine spezifisch abbauenden Proteasomkomplexes wesentliche pathogenetische Bedeutung zukommt.

\section{Eosinophile Polymyositis}

Die eosinophile Polymyositis entsteht als Teilmanifestation eines hypereosinophilen Syndroms, das auch andere Organsysteme betrifft. Sie spricht gut auf Steroidtherapie an.

\section{Fokale Myositis}

Der Begriff sollte den Formen idiopathischer Myositis vorbehalten bleiben, die streng lokalisiert sind, meist auf eine Extremität, und nicht den fokalen Beginn einer DM/PM darstellen. Assoziationen mit Erythemen kommen vor. In manchen Fällen wurde ein erfolgreiches chirurgisches Vorgehen beschrieben.

\section{Orbitale Myositis}

Die idiopathische orbitale Myositis beginnt meist plötzlich und einseitig mit periorbitalen Schmerzen, Diplopie und einer begleitenden Weichteilschwellung. Die Gegenseite kann nach Tagen oder Wochen folgen. Im CT oder MRT kann man die Schwellung einzelner oder mehrerer extraokulärer Muskeln darstellen, die oft eine pathologische Kontrastmittelaufnahme zeigen. Die Krankheit ist steroidsensibel und spricht meist rasch an.

\section{Polymyositis bei HIV-Infektion}

HIV-positive Patienten können eine schleichend auftretende und langsam progrediente Myositis entwickeln, die histopathologisch nicht von einer PM unterschieden werden kann. Diese Myositis kann das erste Symptom der Krankheit sein, entsteht aber häufiger erst im Rahmen eines Aids. Die Kreatinkinase (CK) kann bis auf $1.000 \mathrm{U} / \mathrm{l}$ erhöht sein, und Rhabdomyolyse und Myoglobinurie kommen ebenfalls vor. Die Krankheit spricht in der Regel auf eine Therapie mit Steroiden an. Differenzialdiagnostisch muss eine zidovudininduzierte Myopathie ausgeschlossen werden.

\section{Eosinophile Myofasziitis und Makrophagenmyofasziitis}

Beide Krankheiten führen idiopathisch zu lokalen Schmerzen der betroffenen Körperabschnitte, ödematöser Verschwellung von Faszie und angrenzendem Muskel und meist proximaler Schwäche. Weitere Allgemeinsymptome wie Fieber und Arthralgien kommen vor. Histologisch zeigen die verdickten Faszien eosinophile oder Makrophageninfiltrate. Mindestens eine Form entsteht nach Impfungen mit Präparaten, die Aluminiumhydroxyd enthalten, das eine zytotoxische T-ZellReaktion auslöst. Steroide sind meist gut wirksam.

\section{Literatur}

Dalakas MC (1991) Polymyositis, dermatomyositis and inclusion body myositis. N Engl J Med 325: 1487

Dalakas MC (1995) Update on the use of intravenous immune globulin in the treatment of patients with inflammatory muscle disease. J Clin Immunol 15: 70-75

Dalakas ML, Karpati G (2001) Inflammatory myopathies. In: Karpati G, Hilton-Jones D, Griggs PC (eds) Disorders of voluntary muscle, 7th edn. University Press, Cambridge

Engel AC, Franzini-Armstrong C (2004) Myology, 3rd edn. McGraw-Hill, New York

Gherardi RK, Coquet M, Cherin P et al. (2001) Macrophagic myofasciitis lesions assess long-term persistence of vaccine-derived aluminium hydroxide in muscle. Brain 124: 1821-1831 